\title{
Resultados de la simulación de gasificación del coque de petróleo mexicano de las refinerías de Ciudad Madero y CAdEREYTA
}

\section{RESUITS OF THE SIMULATION OF GASIFICATION OF THE MEXICAN PETROLEUM COKE FROM THE REFINERIES OF CIUdAd MAdERO ANd CAdEREYTA}

\author{
J.C. González-Rocha ${ }^{1,2}$, R. Longoria-Ramírez ${ }^{1}$ y G. Urquiza-Beltrán ${ }^{1,3}$ \\ ${ }^{1}$ Centro Nacional de Investigación y Desarrollo Tecnológico, Departamento de Ingeniería Mecánica, \\ Cuernavaca, Morelos, México, ${ }^{2}$ Instituto Tecnológico de Cd. Madero, \\ División de Estudos de Posgrado e Investigación, Cd. Madero, Tamaulipas, México y \\ ${ }^{3}$ Universidad Autónoma del Estado de Morelos, Centro de Investigación en Ingeniería y Ciencias \\ Aplicadas, División de Turbomaquinaria, Cuernavaca, Morelos, México. \\ E-mails: rlr@cenidet.edu.mx, jcglezr_20@yahoo.com.mx,clemente02@prodigy.net.mx, \\ gurquiza@uaem.mx
}

(Recibido: junio de 2007; aceptado: noviembre de 2007)

\section{Resumen}

En este trabajo se evalúa energéticamente la gasificación del coque de petróleo de las refinerías de Cd. Madero y Cadereyta de México. La evaluación energética del gas sintético (gassin) se centra principalmente en la composición química del gassin limpio (gasl) y sus características energéticas: el poder calorífico, la potencia energética, la eficiencia de gas frío y la eficiencia térmica de gasificación. Los resultados de la simulación indican que la gasificación del coque de Cadereyta denominado CRC, produce un gasl con mejor rendimiento y características energéticas que el gasl obtenido al gasificar el coque de petróleo de Cd. Madero, denominado CRM. Cuando se gasifica el CRC, la razón de producción de gasl obtenida es de $2.07 \mathrm{~kg}_{\text {gas }} / \mathrm{kg}_{\text {coque }}$, mientras que la razón de producción de gasl para el CRM es de $1.89 \mathrm{~kg}_{\text {gas }} / \mathrm{kg}_{\text {coque }}$. El poder calorífico del gasl del CRC es de $12729.56 \mathrm{~kJ} / \mathrm{kg}_{\text {gasl }}$ mientras que para el CRM es de $11639.13 \mathrm{~kJ} / \mathrm{kg}_{\text {gas }}$. La potencia energética del gasl del CRC es mayor con una diferencia de $25.87 \mathrm{GJ} / \mathrm{h}$, con respecto a la potencia energética del gasl del CRM. Con las condiciones de operación especificadas, en la gasificación del coque de petróleo CRC se generan energía a razón de $26.46 \mathrm{MJ} / \mathrm{kg}_{\text {cooue }}$, mientras que al gasificar el coque de petróleo CRM se generan $22.09 \mathrm{MJ} / \mathrm{kg}_{\text {coque }}$. Actualmente, el coque producido en la refinería de Cd. Madero se consume en las plantas de potencia de Tamuín, San Luis Potosí, México, mientras que el coque que produce la refinería de Cadereyta se usa en la industria cementera.

Descriptores: Gasificación, gas sintético, coque del petróleo, regeneración.

\section{Abstract}

This paper evaluates in terms of energy the gasification of the petroleum coke from the refineries of Cd Madero and Cadereyta in Mexico. The energy evaluation of the synthetic gas (syngas) focuses mainly on the chemical composition of the clean syngas (gasl) and its energy characteristics: heating value, the energy power, the cold gas efficiency and the thermal efficiency of gasification. The results of the simulation indicate 
Resultados de la simulación de gasificación del coque de petróleo mexicano de las refinerías de ...

that the gasification of the petroleum coke from Cadereyta called CRC, produces a gasl with better performance and energy characteristics than those obtained when gasifying the coke of petroleum from Cd. Madero, called CRM. When the CRC is gasified, the rate of production of gasl obtained is of $2.07 \mathrm{~kg}_{\text {gas }} / \mathrm{kg}_{\text {coque }}$, while the rate of production of gasl for the CRM is $1.89 \mathrm{~kg}_{\text {gasl }} / \mathrm{kg}_{\text {coque }}$. The HHV of the gasl of the CRC is 12729.56 $\mathrm{kJ} / \mathrm{kg}_{\text {gasl }}$ while for the CRM is $11639.13 \mathrm{~kJ} / \mathrm{kg}_{\text {gasl }}$. The energy power of the gasl of the $C R C$ is greater, with a difference of $25.87 \mathrm{GJ} / \mathrm{h}$, with regard to the energy power of the gasl of the CRM. Under the specified conditions of operation, energy is generated at a rate of $26.46 \mathrm{MJ} / \mathrm{kg}_{\text {coque }}$ coke in the gasification of the CRC coke of petroleum, while when gasifying the CRM coke of petroleum $22.09 \mathrm{MJ} / \mathrm{kg}_{\text {coque }}$ are generated. Currently, the petroleum coke produced in the refinery of Madero is consumed in the power plants of Tamuín, San Luis Potosí, Mexico, while the petroleum coke produced by the refinery of Cadereyta is used in the cement industry.

Keywords: Gasification, synthetic gas, petroleum coke, regeneration.

\section{Introducción}

El objetivo del presente trabajo es encontrar, mediante la simulación del proceso de gasificación, las características energéticas del gas sintético limpio obtenido del coque de petróleo procedente de dos plantas coquizadoras del país, así como las eficiencias térmicas de gasificación $\left(\eta_{t g}\right)$ y las eficiencias de gas frío (EGF).

El conocimiento de estos parámetros resulta de importancia, una vez que son validados mediante el modelo de gasificación utilizado, para posteriormente evaluar la factibilidad y conveniencia de integrar un sistema de gasificación de estos coques a una planta de Ciclo Combinado para la generación de electricidad en el país.

El coque de refinería, en general, es un producto residual sólido de alto contenido energético, alto índice de pulverización, bajo en volátiles, alto contenido de azufre (alrededor de un $7 \%$ ) y metales como el vanadio y níquel.

A nivel mundial, en la última década, se ha incrementado el interés por usar el coque del petróleo como combustible en la generación limpia de energía eléctrica aplicando la tecnología de gasificación (Holt, 1996), (Mahagaokar and Hauser,
1992), (Johnk and Abdulally, 1996), (Generux and Doucette, 1996), (Anthony, et al., 1994), (Anthony et al., 1997), (Swain, 1991), (Yañez, 2000), (Bryan et al., 1996). Asimismo, se han realizado estudios sobre las características cinéticas de la gasificación del coque de petróleo (Hui et al., 2006) y modelos de simulación de la gasificación del coque de petróleo en reactores de lecho fluidizante, analizando las razones de alimentación de oxígeno y vapor con respecto a la alimentación del coque de petróleo (Nagpal et al., 2004). Este interés se debe principalmente al incremento en la producción del coque de petróleo como consecuencia del procesamiento de mayores volúmenes de crudos pesados en las refinerías y al incremento en el rendimiento de los productos con alto valor agregado, como la gasolina, turbosina, diesel o combustóleo, entre otros (González et al., 1998).

En México, se han estimado las posibilidades de generación de energía eléctrica a partir del uso del coque de petróleo, utilizando la tecnología de gasificación (Millán, 1992), (Fernández, 1991), (Bolado, 1995), (Fernández et al., 1993), (Fabela, 1998), (Fernández et al., 1998), (González, 2007). Actualmente, el coque de petróleo producido en la refinería de $\mathrm{Cd}$. Madero es consumido en su totalidad en el complejo termoeléctrico de Tamuín San Luis Potosí, mientras que el coque de 
petróleo que se produce en la refinería de Cadereyta, se ha diversificado a diferentes usos, entre ellos, en la industria cementera.

En el presente estudio se realiza una simulación energética comparativa entre la gasificación del coque de petróleo producido en la refinería de $\mathrm{Cd}$. Madero y la gasificación del coque de petróleo producido en la refinería de Cadereyta, con la finalidad de determinar de cuál de ambos coques se puede producir un gas sintético (gassin) con las mejores propiedades energéticas para ser usado como combustible, sea para generar electricidad limpia o para usarse en una refinería aplicando el concepto de la regeneración (producción de electricidad, vapor y productos químicos) (Northridge, 1986) .

Para la simulación se usó el paquete ASPEN PLUS SHELL (2003) como herramienta complementaria, con el cual se obtuvieron los presentes resultados, eligiéndose este simulador por su gran versatilidad de modelos termodinámicos y su gran flexibilidad para la simulación en interfases con el usuario.

\section{Producción de coque de petróleo en México}

La producción de coque de petróleo en México se ha incrementado en años recientes, debido principalmente a la reconfiguración de las refinerías de Cd. Madero, Tamaulipas y Cadereyta, Nuevo León.

Las siguientes estadísticas conforman una selección de datos obtenidos de las referencias (PEMEX, 2000-2004), (Secretaría de Energía, 2005 a y b), [1], (Aguilar et al., 2000), (Secretaría de Energía 2005 c, 2003, 2001) (IMP, 2002, 2001),
Ios cuales, expresan la tendencia en cuanto a la producción de coque de petróleo durante el período de 2002 a 2006 en México.

Con la integración de la coquizadora en la refinería de Cadereyta, la producción nacional de coque se incrementó de 107.1 toneladas por día (TPD) producidas únicamente por la refinería de Cd. Madero en el 2002 a 2324.36 TPD en el año 2003. En el siguiente año esta producción se incrementó significativamente en un $70 \%$ con respecto al año anterior, ver Tabla 1. Aproximadamente, durante el periodo 2003-2005 por cada 1000 barriles de crudo procesado se produjeron 15.6 toneladas de coque, en promedio.

Se estima que con la reconfiguración de la refinería de Minatitlán iniciada en el 2003 y su entrada en operación para el 2008 y en conjunto, con la reconfiguración del resto de las refinerías (Salamanca, Salina Cruz y Tula), las cuales conforman el Sistema Nacional de Refinación, la producción de coque de petróleo (petcoque) se incremente a $3678.6 \mathrm{mta}$ (miles de toneladas anuales) equivalente a 10218 TPD.

En cuanto a la importación de coque de petróleo, el principal proveedor son los Estados Unidos de América con 1709.9 mta en el año 2002 y con $2166.8 \mathrm{mta}$ en el 2003, esto representó un incremento de 21 \%. Para el año 2006 se estimó un incremento en la importación de coque de $13 \%$ respecto a la importación del año 2003. Este incremento se debió principalmente a la demanda de la industria cementera.

Tabla 1. Producción nacional de coque en las refinerías de Cadereyta y Cd. Madero (TPD)

\begin{tabular}{cccccc}
\hline Refinería/año & 2002 & 2003 & 2004 & 2005 & 2006 \\
\hline Madero & 107.10 & 1234.50 & 1926.90 & 1976.71 & 1791.70 \\
Cadereyta & 0 & 1089.86 & 2026.60 & 32.77 & 2138.61 \\
Total & 107.10 & 2324.36 & 3953.50 & 3768.40 & 4561.39 \\
\hline
\end{tabular}


Resultados de la simulación de gasificación del coque de petróleo mexicano de las refinerías de ...

\section{Consumo de coque de petróleo en México}

El coque de petróleo producido en la refinería de $\mathrm{Cd}$. Madero se consume en conjunto con el coque de petróleo importado de la refinería de Houston para generar una potencia eléctrica de $460 \mathrm{MW}$ mediante el complejo termoeléctrico de Tamuín, San Luis Potosí (2 unidades de $230 \mathrm{MW}$ ). El coque de petróleo consumido por cada unidad se encuentra entre 1800 y 2200 TPD. De acuerdo con este consumo y con el dato de la producción de coque de petróleo de la refinería de $\mathrm{Cd}$. Madero dado en la tabla 2 para el período 2004-2005, es posible estimar lo siguiente: con el coque de petróleo de la refinería de Cd. Madero se están generando aproximadamente $230 \mathrm{MW}$ con una razón de producción de energía de $10 \mathrm{MJ} / \mathrm{kg}_{\text {coque. }}$

Con respecto al coque de petróleo producido en la refinería de Cadereyta éste está siendo utilizado fundamentalmente en la industria del cemento (CEMEX).

\section{Propiedades del coque de petróleo mexicano}

De acuerdo a las cantidades de coque de petróleo que se están produciendo en las refinerías mexicanas de Cd. Madero y Cadereyta y a su gran potencial comercial, se ha seleccionado al coque de petróleo producido por estas refinerías con la finalidad de determinar el potencial energético del gassin obtenido en un proceso de gasificación.

El contenido de cenizas es importante, primeramente por el efecto que éstas pueden tener en el interior del gasificador en cuanto a la transferencia de calor que impacta directamente en la eficiencia de gas frío y en la eficiencia térmica de gasificación.

El bajo contenido de materia volátil (alrededor 10\%) comparado con el del carbón, contribuye a su baja reactividad, lo cual es compensado por el alto contenido de oxígeno e hidrógeno, y que pueden incrementarse con el vapor de agua que se integra al proceso de gasificación.
El alto contenido de azufre tiene como consecuencias, primeramente, la influencia de éste en el mecanismo de corrosión sobre los componentes del sistema de gasificación, y segundo, la necesidad de considerar un proceso adicional integrado al sistema de gasificación para su eliminación del gassin crudo (gasc).

El análisis próximo (tal como se recibe), el análisis último (libre de humedad) y el poder calorífico superior del coque de petróleo ( PCS $_{\text {coque }}$ ) producido en las refinerías de Cd. Madero y Cadereyta y las normas que se aplicaron para obtener sus valores se presentan en la tabla 2 .

\section{Mercado potencial del coque del petróleo}

La gasificación es una tecnología que en la última década ha resultado de gran aceptación para convertir combustibles sólidos como el carbón, biomasa y últimamente el coque de refinería en un gas sintético. Básicamente consiste en convertir un combustible sólido en un gas limpio, libre hasta en un $99 \%$ de compuestos de azufre $\left(\mathrm{H}_{2} \mathrm{~S}, \mathrm{COS}\right)$ y de metales pesados como vanadio y níquel.

La reacción básica de gasificación puede expresarse como sigue;

$$
\mathrm{C}_{n} \mathrm{H}_{m}+n / 2 \mathrm{O}_{2} \Rightarrow n \mathrm{CO}+m / 2 \mathrm{H}_{2}
$$

La composición del gas resultante (gassin) está formado principalmente de $\mathrm{CO}$ y $\mathrm{H}_{2}$ (alrededor de $90 \%$ ) y de $\mathrm{N}_{2}, \mathrm{CO}_{2}, \mathrm{H}_{2} \mathrm{O}, \mathrm{H}_{2} \mathrm{~S}$ y COS.

Entre las principales compañías a nivel mundial que usan la tecnología de gasificación se puede mencionar a Texaco, Shell, Lurgi y BGL con aplicaciones en países como Estados Unidos de América, Holanda, España, Italia, Japón, India, Sudáfrica, entre otros. Desde 1986 las experiencias de gasificación, exclusivamente de coque de petróleo, se han usado para producir hidrógeno, vapor y electricidad (Tabla 3). En esta tabla se puede observar la tendencia en el uso cada vez más continuo del coque de petróleo (petcoque). 
DOI: http://dx.doi.org/10.22201/fi.25940732e.2008.09n2.009

J.C. GOnzález-ROCha, R. LONGoria-Ramírez y G. Urquiza-Beltrán

Tabla 2. Composición del coque CRM y CRC, (\% peso)

(Centro Nacional de Investigación y Desarrollo Tecnológico, 2005)

\begin{tabular}{cccc}
\hline Análisis próximo & CRM & CRC & Norma \\
\hline Humedad & 10.54 & 1.21 & D3173-03 \\
Cenizas & 0.35 & 0.36 & D3174-02 \\
Materia volátil & 10.22 & 10.65 & D3175-02 \\
Carbón fijo & 78.89 & 87.78 & D3172-R02 \\
\hline & & & \\
\hline Análisis último & & & D3174-02 \\
Cenizas & 0.35 & 0.36 & D5373-02 \\
Carbono & 84.24 & 84.63 & D5373-02 \\
Hidrógeno & 3.90 & 3.91 & D5373-02 \\
Nitrógeno & 0.79 & 0.72 & D129-00 \\
Azufre & 6.04 & 5.91 & \\
Oxígeno & 4.68 & 4.47 & D5865-03 \\
PCS & & & \\
coque $(\mathbf{k J} / \mathbf{k g})$ & 35202.61 & 38987.48 & \\
\hline
\end{tabular}

Tabla 3. Experiencia de gasificación exclusivamente de coque de petróleo (Fabela, 1998), (Ahman et al., 2002), (EPRI, 2006)

\begin{tabular}{ccccc}
\hline Lugar & Combustible & (TPD) & Producción & Año \\
\hline Japón & Petcoque & 1650 & $\mathrm{H}_{2}$ y electricidad & 1986 \\
Taiwan & Petcoque & ---- & Electricidad & 1993 \\
E.U.A. & Petcoque & 5450 & Electricidad y vapor & 1996 \\
Holanda & Petcoque & 2350 & Electricidad & 1998 \\
España & Petcoque & 2590 & Electricidad & 2000 \\
México & Petcoque & 2200 & Electricidad & 2002 \\
India & Petcoque & 2300 & Electricidad & 2006 \\
Italia & Petcoque & 5000 & Electricidad, vapor, químicos & 2006 \\
\hline
\end{tabular}

\section{Modelo termodinámico de gasificación}

El modelo termodinámico utilizado fue el de Mahagaokar y Huser (1992), el cual fue validado para su aplicación. En este trabajo de simulación del proceso de gasificación de coque procedente de refinerías de petróleo mexicano se consideró una temperatura de $1515^{\circ} \mathrm{C}$ y presión de 40 bar. De acuerdo con los resultados de la simulación, el efecto de la presión resultó ser de muy limitada influencia, en tanto que para la temperatura se tomó en cuenta que la velocidad de reacción, para las reacciones de combustión que se dan más adelante, son limitadas por la transferencia de masa a 
Resultados de la simulación de gasificación del coque de petróleo mexicano de las refinerías de ...

temperaturas mayores de $1150{ }^{\circ} \mathrm{C}$ no así la cinética de gasificación de vapor de agua y dióxido de carbono a temperaturas mayores a $1400{ }^{\circ} \mathrm{C}$ (Hui, 2006).

En particular, la reacción de equilibrio del CO con vapor de agua, ecuación 7 , es una reacción importante, si se considera que interviene en el control de la composición del gassin limpio (gasl); a temperaturas mayores de $1000^{\circ} \mathrm{C}$ su velocidad de reacción es tan rápida que puede considerarse que se encuentra en equilibrio (Hui, 2006), (Nagpal et al., 2004), como es el caso de este trabajo de simulación.

Durante un proceso de gasificación se desarrollan una serie de reacciones químicas que dependen de las condiciones de operación del gasificador. Las principales reacciones químicas involucran al carbono, monóxido de carbono, dióxido de carbono, hidrógeno, vapor de agua, metano, ácido sulfhídrico y sulfuro de carbonilo. Estas reacciones pueden expresarse de la siguiente manera (Himan et al., 2003):

$$
\begin{array}{llll}
2 \mathrm{C} & \mathrm{O}_{2} & 2 \mathrm{CO}-111 \mathrm{~kJ} / \mathrm{mol} \\
2 \mathrm{CO} & \mathrm{O}_{2} & 2 \mathrm{CO}_{2}-393 \mathrm{~kJ} / \mathrm{mol} \\
2 \mathrm{H}_{2} & \mathrm{O}_{2} & 2 \mathrm{H}_{2} \mathrm{O}-242 \mathrm{~kJ} / \mathrm{mol} \\
\mathrm{C} & \mathrm{CO}_{2} & 2 \mathrm{CO}+172 \mathrm{~kJ} / \mathrm{mol} \\
\mathrm{C} & \mathrm{H}_{2} \mathrm{O} & \mathrm{CO} & \mathrm{H}_{2}+131 \mathrm{~kJ} / \mathrm{mol} \\
\mathrm{C} & 2 \mathrm{H}_{2} & \mathrm{CH}_{4}-75 \mathrm{~kJ} / \mathrm{mol}
\end{array}
$$

Esencialmente las ecuaciones 1, 4, 5 y 6 representan reacciones heterogéneas que determinan la composición del gassin en el estado de equilibrio. Estas cuatro reacciones describen la manera en que el coque de petróleo puede ser gasificado. La reacción 4 produce el $\mathrm{CO}$ al gasificar el carbono en una atmósfera de $\mathrm{CO}_{2}$, la reacción 5 es predominante sobre el proceso gas-agua y la reacción 6 es la base de hidrogenación en el proceso de gasificación. Obsérvese que en las ecuaciones 4 y 6 se encuentran involucradas las reacciones homogéneas del $\mathrm{CO}$ y la reacción de metanación con vapor de agua (7) y (8).

$$
\begin{array}{llll}
\mathrm{CO} & \mathrm{H}_{2} \mathrm{O} & \mathrm{CO}_{2} & \mathrm{H}_{2}-41 \mathrm{~kJ} / \mathrm{mol} \\
\mathrm{CH}_{4} & \mathrm{H}_{2} \mathrm{O} & \mathrm{CO}_{2} & 3 \mathrm{H}_{2}+206 \mathrm{~kJ} / \mathrm{mol}
\end{array}
$$

Las ecuaciones 4 a 8 son reacciones reversibles que se realizan simultáneamente a velocidades diferentes.

En general, toda mezcla reactiva a temperatura y presión determinada se desarrolla en la dirección del decrecimiento de la función de Gibbs (G) bajo el siguiente criterio de equilibrio químico (Cengel et al., 1998), (Bejan, 1980):

$$
(d G)_{T, P} \quad 0
$$

Es decir, cuando la función de Gibbs es mínima la reacción química para y se establece el equilibrio químico. Es necesario especificar que una reacción química a una temperatura y presión constantes no puede desarrollarse en dirección del incremento de la función de Gibbs, debido a que se violaría la segunda ley de la termodinámica

$$
d S \quad \frac{Q}{T}
$$

Al resolver para cada reacción desde la 4 a 6 involucrando la 7 y 8 se puede obtener el criterio de equilibrio químico siguiente:

$$
v_{i} \bar{g} \quad v_{i} \bar{g} \quad 0
$$

Donde:

$v \quad$ es el coeficiente estequiométrico.

$\bar{g} \quad$ es la función de Gibbs molar.

p representa a los productos de gasificación. 
r representa a los reactivos involucrados.

i iesimo producto o reactivo en la reacción química.

Las constantes de equilibrio $(K)$ de las reacciones involucradas en la gasificación se obtuvieron usando la correlación

$$
\operatorname{Ln}\left(K_{p, T}\right) \quad \operatorname{Ln}\left(k_{p, T_{0}}\right) \quad f(T)
$$

Considerando la ecuación 12 es posible encontrar las fracciones molares en el estado de equilibrio del gasl obtenido mediante la gasificación del coque de petróleo mexicano (Himan et al., 2003).

En la figura 1 se muestra esquemáticamente el modelo usado para el proceso de gasificación del coque de petróleo.

\section{Resultados y discusión}

La evaluación energética del gas sintético (gassin) se centra principalmente en la obtención de la composición química del gassin limpio (gasl) y sus características energéticas: el poder calorífico superior $\left(\mathrm{PCS}_{\text {gas }}\right)$, la potencia energética $\left(\Omega_{\text {gas }}\right)$, eficiencia de gas frío $\left(\mathrm{EGF}_{\text {gas }}\right)$ y la eficiencia térmica de gasificación $\eta_{\operatorname{tg}}$.
El sistema de gasificación se validó comparando los resultados obtenidos de la literatura (Mahagaokar et al., 1992) y considerando iguales condiciones de operación en la gasificación del coque mexicano en un proceso de gasificación Shell.

\section{Estimación de la composición del gassin limpio (gasl)}

Una vez que es de interés la composición química y las características energéticas del gassin limpio (gasl), es decir, el producido en el sistema de gasificación, todos los resultados se referirán respecto al gasl. Estos resultados se obtuvieron para una relación másica oxígeno/coque de 1.02, una alimentación de oxígeno de $6036.388 \mathrm{~kg} / \mathrm{h}$, una relación vapor de agua/oxígeno de 23 \% y una presión y temperatura de gasificación de 42 bar y $1515^{\circ} \mathrm{C}$, respectivamente. El poder calorífico del gassin fue calculado con las entalpías de reacción respectivas del CO, $\mathrm{H}_{2}$ y $\mathrm{H}_{2} \mathrm{~S}$ (Cengel et al., 1998), (Bejan, 1980). En la tabla 4 se muestran la composición química del gassin limpio (gasl) obtenida de la gasificación de los coques CRC y CRM. Como se puede observar, alrededor del $90 \%$ del gasl está compuesto por $\mathrm{CO}$ y $\mathrm{H}_{2}$, con trazas de $\mathrm{CO}_{2}, \mathrm{~N}_{2}, \mathrm{CH}_{4}$, $\mathrm{H}_{2} \mathrm{~S}$, COS, y $\mathrm{H}_{2} \mathrm{O}$.

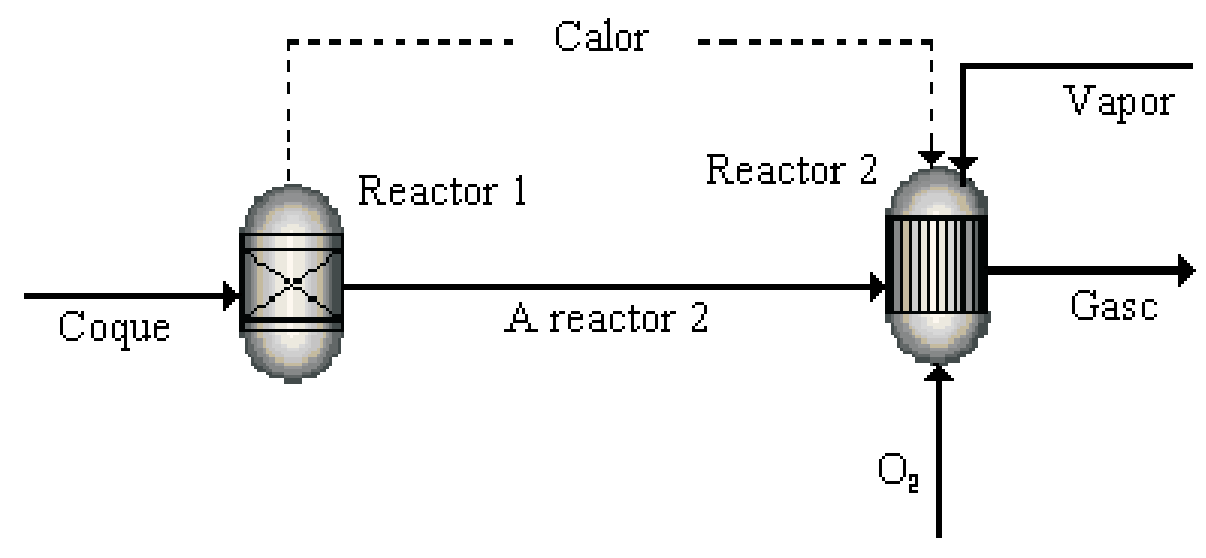


Resultados de la simulación de gasificación del coque de petróleo mexicano de las refinerías de ...

El poder calorífico superior del gasl $\left(\mathrm{PCS}_{\text {gasl }}\right)$ obtenido para el CRM es de $11639.19 \mathrm{~kJ} / \mathrm{kg}_{\text {gasl }} \mathrm{y}$ para el CRC es de $12729.56 \mathrm{~kJ} / \mathrm{kg}_{\text {gasl }}$ ambos difieren en aproximadamente $1090.429 \mathrm{~kJ} / \mathrm{kg}_{\text {gas }}$ equivalente en un $9.4 \%$ mayor para el CRC. Este resultado se debe principalmente a la diferencia entre ambos coques en cuanto al contenido de carbón fijo (CF) y humedad (H), ver tabla 2 y al mayor contenido de $\mathrm{CO}$ en el gasl del CRC (Tabla 4).

\section{Eficiencia del gas frío del gasl (EGF)}

La eficiencia de gas frío del gasl es un parámetro de rendimiento que expresa el porcentaje de energía química del coque de petróleo contenida en el gasl y se define como:

$$
E G F_{\text {gasl }} \frac{\Omega_{\text {gasl }}(G J / h)}{\Omega_{\text {coque }}(G J / h)} 100
$$

Considerando los resultados de la tabla 5 , se puede observar que un incremento en el contenido de carbón fijo con un decremento en la humedad, tiene como consecuencia un incremento en el porcentaje molar del $\mathrm{CO}$ en el gasl; así como también un incremento en el poder calorífico superior $\left(P_{\text {CS }}\right)$ y en la potencia energética del gasl $\left(\Omega_{\text {gas }}\right)$. De igual manera, en la eficiencia de gas frío del gasl $\left(E_{\text {gas }}\right)$, esto de acuerdo con la definición de $E_{\text {gas }}$ (Ahman et al., 2002). Comparando ambos resultados se tiene que la $\mathrm{EGF}_{\text {gasl }}$ del $\mathrm{CRC}$ es de
67.87 \% y para el CRM de $62.75 \%$ con una diferencia aproximada de 5 puntos porcentuales.

\section{Eficiencia térmica de gasificación $\left(\eta_{t g}\right)$}

La eficiencia térmica de gasificación se define como la relación entre la suma de la potencia energética del gassin crudo $\left(\Omega_{\text {gasc }}\right)$ y la potencia energética contenida en el vapor generado $\left(\Omega_{\text {vapor }}\right)$, respecto a la potencia energética del coque de petróleo alimentado $\left(\Omega_{\text {coque }}\right)$, expresando todas las cantidades en GJ/h. Prácticamente, el vapor se genera en la sección de enfriamiento al intercambiar energía calorífica entre el gassin crudo (gasc) que sale del reactor y el agua de alimentación de la sección de enfriamiento.

En la tabla 6 se puede observar específicamente, la potencia energética del gasc $\left(\Omega_{\text {gasc }}\right)$, la potencia energética del vapor $\left(\Omega_{\text {vapor }}\right)$ y la potencia energética del coque alimentado $\left(\Omega_{\text {coque }}\right)$. En la gasificación del CRC y del CRM la energía recuperada en forma de vapor es de alrededor de $7.5 \%$ para ambos, sin embargo, la potencia energética del gasc difiere notablemente, siendo mayor la potencia energética del gasc del CRC en una cantidad de $27 \mathrm{GJ} / \mathrm{h}$ respecto a la potencia energética del CRM. Esta diferencia entre las potencias energéticas de cada gasc, hace posible que la eficiencia térmica obtenida $\left(\eta_{\mathrm{tg}}\right)$ al gasificar el CRC sea de $83.65 \%$ y la eficiencia térmica obtenida al gasificar el CRM tenga un valor de $79.66 \%$.

Tabla 4. Composición del gasl como producto de la gasificación del coque de refinería mexicano (CRM y CRC), porcentaje molar

\begin{tabular}{ccc}
\hline & CRM & CRC \\
\hline Componente & Gassin limpio & Gassin limpio \\
$\mathrm{CO}$ & 64.075 & 68.037 \\
$\mathrm{H}_{2}$ & 27.008 & 27.645 \\
$\mathrm{CO}_{2}$ & 6.662 & 2.292 \\
$\mathrm{~N}_{2}$ & 0.797 & 0.703 \\
$\mathrm{CH}_{4}$ & 0.003 & 0.012 \\
$\mathrm{H}_{2} \mathrm{COS}$ & 0.108 & 0.125 \\
$\mathrm{H}_{2} \mathrm{O}$ & $8.3236 \mathrm{e}-03$ & $8.4322 \mathrm{e}-03$ \\
\hline
\end{tabular}


DOI: http://dx.doi.org/10.22201/fi.25940732e.2008.09n2.009

J.C. González-Rocha, R. Longoria-Ramírez y G. Urquiza-Beltrán

Tabla 5. Efecto de la variación del carbono fijo y la humedad sobre el PCS gasl, la $\Omega_{\text {gasl }}$ y la EGF gasl

\begin{tabular}{|c|c|c|c|c|c|}
\hline & \multicolumn{5}{|c|}{ CRM } \\
\hline $\begin{array}{c}\text { CF } \\
\% \text { peso }\end{array}$ & $\begin{array}{c}\mathrm{H} \\
\% \text { peso }\end{array}$ & $\begin{array}{c}\mathrm{co} \\
\% \mathrm{~mol}\end{array}$ & $\begin{array}{l}\mathrm{PCS}_{\text {gasl }} \\
\mathrm{kJ} / \mathrm{kg}_{\text {gas }}\end{array}$ & $\begin{array}{l}\Omega_{\text {gasl }} \\
\mathrm{GJ} / \mathrm{h}\end{array}$ & $\begin{array}{l}\text { EGF } \\
(\%)\end{array}$ \\
\hline 73.89 & 15.54 & 61.80 & 11052.95 & 117.64 & 56.46 \\
\hline 78.89 & 10.54 & 64.08 & 11639.14 & 130.74 & 62.75 \\
\hline 83.89 & 5.54 & 66.22 & 12197.43 & 143.84 & 69.04 \\
\hline 88.89 & 0.54 & 68.25 & 12729.79 & 156.92 & 75.32 \\
\hline
\end{tabular}

\begin{tabular}{cccccc}
\hline & \multicolumn{3}{c}{ CRC } \\
\hline $\begin{array}{c}\text { CF } \\
\% \text { peso }\end{array}$ & $\begin{array}{c}\mathrm{H} \\
\% \text { peso }\end{array}$ & $\begin{array}{c}\mathrm{CO} \\
\% \mathrm{~mol}\end{array}$ & $\begin{array}{c}\mathrm{PCS}_{\text {gasl }} \\
\mathrm{kJ} / \mathrm{kg}_{\text {gasl }}\end{array}$ & $\begin{array}{c}\Omega_{\text {gasl }} \\
\mathrm{GJ} / \mathrm{h}\end{array}$ & $\begin{array}{c}\mathrm{EGF}_{\text {gasl }} \\
(\%)\end{array}$ \\
\hline 73.78 & 15.21 & 62.056 & 11162.584 & 119.744 & 51.896 \\
78.78 & 10.21 & 64.305 & 11746.796 & 132.921 & 57.606 \\
83.78 & 5.21 & 66.422 & 12303.243 & 146.095 & 63.316 \\
87.78 & 1.21 & 68.037 & 12729.568 & 156.611 & 67.873 \\
88.78 & 0.21 & 68.431 & 12833.204 & 159.229 & 69.008 \\
\hline
\end{tabular}

Tabla 6. Resumen de los resultados obtenidos de la gasificación del coque de refinerías mexicanas; CRM y CRC

\begin{tabular}{|c|c|c|c|c|}
\hline & $\begin{array}{c}\text { Coque } \\
\text { CRM }\end{array}$ & $\begin{array}{c}\text { Coque } \\
\text { CRC }\end{array}$ & $\begin{array}{c}\text { Gasl del } \\
\text { CRM }\end{array}$ & Gasl del CRO \\
\hline PCS $\left(\mathrm{kJ} / \mathrm{kg}_{\text {coque }}\right)$ & 35202.61 & 38987.48 & & \\
\hline $\operatorname{PCS}\left(\mathrm{kJ} / \mathrm{kg}_{\text {gas }}\right)$ & & & 11639.14 & 12729.57 \\
\hline m\&(kg/h) & 5918.30 & 5918.30 & 11232.86 & 12302.96 \\
\hline$\Omega_{\text {coque }}(\mathrm{GJ} / \mathrm{h})$ & 208.340 & 230.740 & & \\
\hline$\Omega_{\text {gasl }}(\mathrm{GJ} / \mathrm{h})$ & & & 130.74 & 156.61 \\
\hline $\mathrm{EGF}_{\text {gasl }}(\%)$ & & & 62.75 & 67.87 \\
\hline$\Omega_{\text {gasc }}(\mathrm{GJ} / \mathrm{h})$ & & & 150.72 & 177.82 \\
\hline$\Omega_{\text {vapor }}(\mathrm{GJ} / \mathrm{h})$ & & & 15.25 & 15.19 \\
\hline $\mathrm{EGF}_{\text {gasc }}(\%)$ & & & 72.34 & 77.07 \\
\hline$\eta_{\operatorname{tg}}(\%)$ & & & 79.66 & 83.65 \\
\hline
\end{tabular}


Resultados de la simulación de gasificación del coque de petróleo mexicano de las refinerías de ...

En la misma tabla 6 se puede observar que, manteniendo el sistema de gasificación operando en estado estable, en la gasificación del CRM, el gasl se produce a razón de $1.89 \mathrm{~kg}_{\text {gas }} / \mathrm{kg}_{\text {coque }}$, mientras que para la gasificación del CRC, el gasl se produce a razón de $2.07 \mathrm{~kg}_{\text {gas }} / \mathrm{kg}_{\text {coque }}$. La potencia energética del gasl del CRC es mayor en 25.87 $\mathrm{GJ} / \mathrm{h}$ respecto a la potencia energética del gasl del $\mathrm{CRM}$, representando un incremento de aproximadamente 4.4 MJ por kg de coque gasi- ficado.

Considerando la potencia energética del gasl $\left(\Omega_{\text {gas }}\right)$ respectiva y la alimentación de coque al reactor de $5918.30 \mathrm{~kg} / \mathrm{h}$, se puede observar lo siguiente; cuando se gasifica el CRM se produce $22.09 \mathrm{MJ} / \mathrm{kg}_{\text {coque }}$, mientras que cuando se gasifica el CRC se produce $26.46 \mathrm{MJ} / \mathrm{kg}_{\text {coque }}$, con lo que es posible generar directamente desde el gasl producido $36.31 \mathrm{MW}$ y $43.50 \mathrm{MW}$, respectivamente. Ambos resultados se obtuvieron con igual alimentación de coque (Tabla 6).

Otro parámetro que comúnmente se usa para definir el desempeño de un proceso de gasificación es la eficiencia de gas frío del gassin crudo $\left(E_{\text {gasc }}\right)$, la cual se define como la relación de la potencia energética del gassin crudo $\Omega_{\text {gasc }}(G J / h)$, respecto a la potencia energética del coque alimentado $\Omega_{\text {coque }}(\mathrm{GJ} / \mathrm{h}$ ) (Himan et al., 2003):

$$
E G F_{\text {crudo }} \frac{\Omega_{\text {gasc }}(G J / h)}{\Omega_{\text {coque }}(G J / h)} \quad 100
$$

Considerando los valores de la tabla 6 que por definición le corresponden a la $\mathrm{EGF}_{\text {gasc }}$ del CRM y CRC, se obtienen valores de $72.34 \%$ y $77.07 \%$, respectivamente. Estos valores son también de gran importancia debido a que indican dos cosas: la cantidad de energía contenida en el gasc inmediatamente después del proceso de gasificación y directamente el grado de pérdida de energía en el reactor.

Considerando que el proceso de gasificación de este estudio es muy similar al utilizado en un gasificador de flujo por arrastre, autores como Mahagaokar y Huser (1992), Nagpal et al., (2004) han reportado similares resultados, principalmente en la $\mathrm{EGF}_{\text {gasc }}$ de $78.6 \%$ y $78.9 \%$, respectivamente. Esta diferencia de debe principalmente, a una diferente relación $\mathrm{H}_{2} / \mathrm{CO}$ obtenida. En la figura 2 se presenta el balance térmico del proceso de gasificación del CRM y CRC.

En esta figura se puede observar que la mayor pérdida de energía se encuentra durante el proceso de gasificación, representando una reducción en el gasc del $28.61 \%$ respecto a la potencia energética del coque CRM alimentado, mientras que en la gasificación del CRC la reducción en el gasc representa un $22.93 \%$ con relación a la potencia energética del coque CRC alimentado. Cabe hacer mención que estas pérdidas no son recuperables como es el caso del calor contenido en el vapor radiactivo producido en el reactor.

\section{Conclusiones}

Los resultados de la simulación indican que la gasificación del coque de Cadereyta produce un gasl con mejores características energéticas comparado con el gasl obtenido al gasificar el coque de petróleo de Cd. Madero.

La razón de producción de gasl por kg de coque alimentado del CRC es de $2.07 \mathrm{~kg}_{\text {gas }} / \mathrm{kg}_{\text {coque }}$, mayor que la razón de producción de gasl del CRM con $1.89 \mathrm{~kg}$ gas $/ \mathrm{kg}_{\text {coque }}$.

Para una igual alimentación de coque de $5918.304 \mathrm{~kg} / \mathrm{h}$ es posible generar 43.5 MW gasificando el coque CRC, mientras que gasificando el coque CRM es posible generar 36.31 MW, con una razón de producción de energía de $26.46 \mathrm{MJ} / \mathrm{kg}_{\text {coque }} \mathrm{y}$ $22.09 \mathrm{MJ} / \mathrm{kg}_{\text {coque }}$, respectivamente.

Las diferencias entre los resultados de la gasificación del coque de petróleo CRM y CRC, se deben a sus contenidos de carbón fijo (CF) y humedad $(H)$, ya que éstos modifican la composición del gasl. 

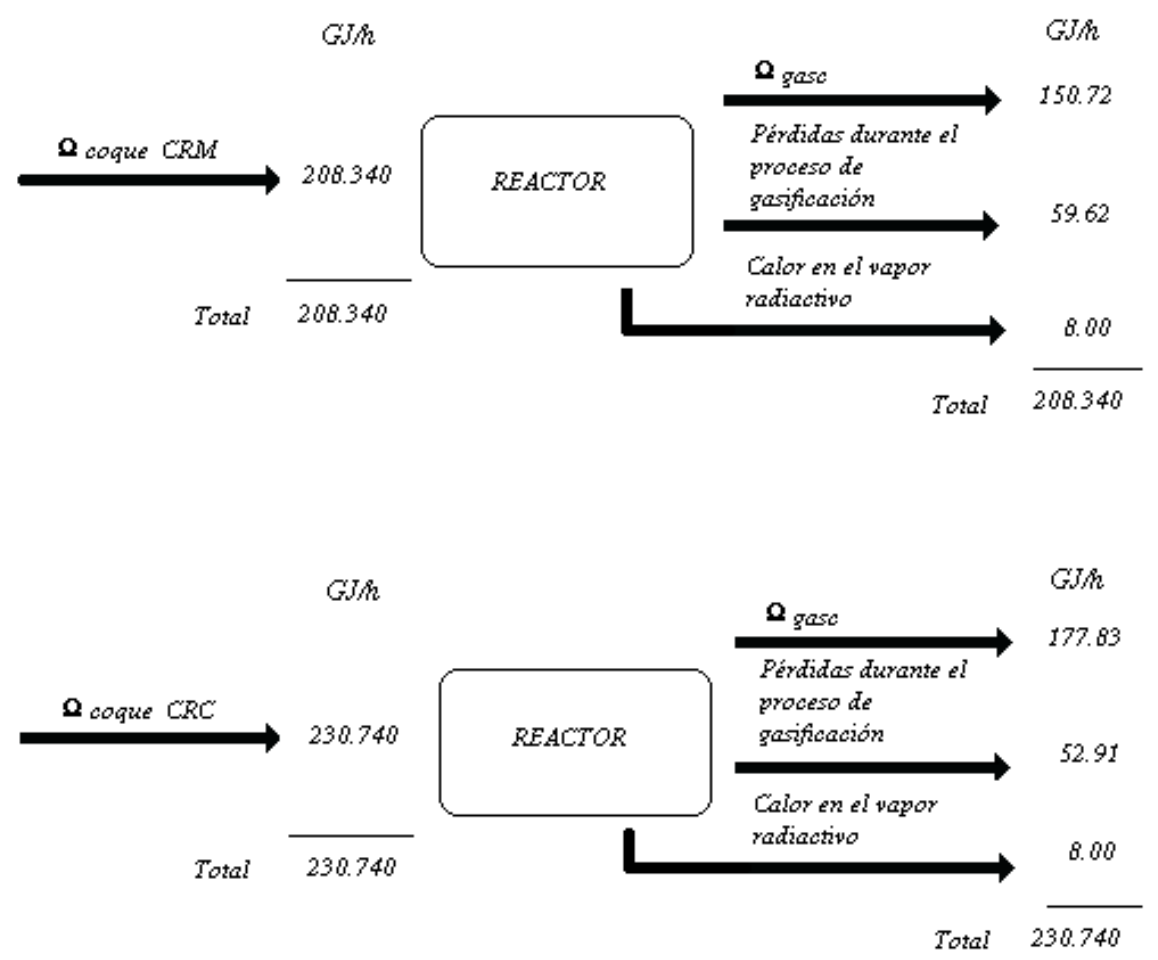

Figura 2. Balance térmico para el proceso de la gasificación del coque CRM y CRC

Por lo anterior, el coque de la refinería de Cadereyta ofrece mejor rendimiento energético que el coque de la refinería de $\mathrm{Cd}$. Madero, en condiciones iguales de operación del sistema de gasificación.

Con el gassin limpio (gasl) obtenido en un sistema de gasificación se puede aplicar el concepto de la trigeneración, haciendo posible generar electricidad (Northridge, 1986), vapor o productos químicos como el $\mathrm{H}_{2}$.

Considerando la razón de producción de energía para el CRM de $22.09 \mathrm{MJ} / \mathrm{kg}_{\text {coque }}$ obtenido como resultado de este estudio y la producción actual de coque de la refinería Madero, se estima que sería posible obtener una generación de electricidad de aproximadamente $500 \mathrm{MW}$.

Finalmente, las simulaciones suministran información detallada y completa relacionada con balances de masa y energía, usualmente con mayor resolución y flexibilidad que las logradas con los trabajos experimentales.

\section{Agradecimientos}

Un especial agradecimiento al CONACYT, al Centro Nacional de Investigación y Desarrollo Tecnológico (cenidet), al Instituto Tecnológico de Cd. Victoria y al Instituto Tecnológico de $\mathrm{Cd}$. Madero por las facilidades otorgadas para la realización de esta investigación.

\section{Referencias}

Aguilar-Rodríguez E. y Cano-Domínguez J.L. Situación actual y perspectivas de las tecnologías para la refinación del petróleo. Instituto Mexicano del Petróleo (IMP), mayo 2000.

Ahman S., Pisano-Altom J., Tsiou C.G. Refinería de Taiwan utilizará coque de petróleo en dos CFB's de avanzada tecnología. Power, mayo/ junio 2002. 
Resultados de la simulación de gasificación del coque de petróleo mexicano de las refinerías de ...

Anthony E. J., Laub I. I. and Smith B. Ignition of coal and coke mixtures in a bench scale fluid bed reactor. CANMET ERL-94-01 (TR), January, 1994.

Anthony E. J., Anderson K., Carson R. and Lau I. I. Petroleum coke and coal start up testing in bubbling fluidized bed combustor. Transaction of The ASME, Journal of Energy Resources Technology, (119):96-109. June, 1997.

ASPEN PLUS SHELL, 2003, Aspen Technology Inc. Pemex refinación [en línea]. Proceso de petróleo crudo por refinería 2000-2004. Disponible en: www.ref.pemex.com.

Bejan A. Advanced Engineering Thermodinamics. Wiley-Interscience publication. John Wiley and Son. 1980.

Bolado-Estadía R. Nuevas tecnologías para la utilización de combustibles fósiles en la generación eléctrica. Publicación Interna, Instituto de Investigaciones Eléctricas (IIE), Cuernavaca, México, pp. 1-41. 1995.

Bryan S., Bock H.Y. and Rosenquist W.A. Petroleum coke as a viable alternative fuel. POWERGEN International, Orlando, Florida, EUA, 1996, pp. 259-267.

Cengel-Yunus A. y Boles-Michael A. Thermodinamics: An Engineering Approach. 3a. Edición, McGraw-Hill. ISBN 0-07-11524-4. 1998.

Centro Nacional de Investigación y Desarrollo Tecnológico-Cenidet. Informe No. 7, Análisis de las muestras de coque procedentes de $\mathrm{Cd}$. Madero, Tamaulipas y Cadereyta, Nuevo león. Ingeniería Mecánica-procesos térmicos, Cuernavaca, Morelos, Marzo 14 del 2005.

EPRI, final report 2006 [en línea]. Petcoke and Low-Rank Coal/Lignite Supply Outlook for IGCC Evaluations. Program 066.0 Future Coal Generation Options, 1013038 Final Report 200 602. Disponible en: http://www.epri.com/Orde rableitemDesc.asp?product_id $=0000000000$ $01013038 \&$ searchdate $=0 \overline{2} / 22 / 2006$.

Fabela R. Alternativas para la utilización del coque. Instituto de Investigaciones Eléctricas (IIE), workshop de carbón, Subdirección de Planeación de Pemex-Refinación, febrero, 1998.
Fernández-Montiel M. y Alaniz-Chávez F.J. Gasificación de combustibles sólidos y de combustóleo para la generación eléctrica. Reporte del estado actual de las tecnologías de gasificación aplicadas a la generación de energía eléctrica, reporte No. 44207, IIE, División de sistemas mecánicos, Departamento de Ingeniería Mecánica, 1993.

Fernández-Montiel M., González-Santaló J.M., Gutiérrez-Ramírez R., Milán-Foressi J. y RomoMillares C. Tendencias tecnológicas en los procesos de combustión en la generación de electricidad. Boletín IIE. Mayo/junio 1998.

Fernández-Montiel M. Procesos de gasificación aplicados a sistemas de cogeneración con residuales sólidos nacionales. Instituto de Investigaciones Eléctricas, CONAE. pp. 401-408. 1991.

Generux R.P. and Doucette B. Pet-Coke-Firing experience envolves over three decades. Power, 7(140):61-62. July/august, 1996.

González-Fernández R., Villalobos H. Procesamiento de crudos pesados en México [en línea]. Instituto Mexicano del Petróleo (IMP), 2001. Disponible en: www.imiq.org/leon 99/memorias/ trabajos/Jt8/jt8-4.htm.

González Rocha J.C. Estudio del desempeño de una planta de potencia de ciclo combinado con gasificación integrada. Tesis Doctoral. Centro Nacional de Investigación y Desarrollo Tecnológico. Departamento de Ingeniería Mecánica, 2007.

Himan C., and Vander-Burgt M. Gasification. Editorial Elsevier Science USA. British Library Cataloguing-in-Publication Data. ISBNO-7506-77 07-4. 2003.

Hui-Zou J. et al. Modeling reactor kinetics of petroleum coke gasification with $\mathrm{CO}_{2}$. Chemical Engineering and Processing, 2006, doi:10.1016/ j.cep.2006.08.008.

Holt N. Gasificación de combustibles fósiles. Boletín Instituto de Investigaciones Eléctricas (IIE), tendencias tecnológicas. Pp. 256-259. Noviembre-Diciembre, 1996.

Instituto Mexicano del Petróleo (IMP) [en línea]. Modelo de optimización del sector eléctrico nacional, 2002. Disponible en: www.imp.mx. 
DOI: http://dx.doi.org/10.22201/fi.25940732e.2008.09n2.009

J.C. González-Rocha, R. Longoria-Ramírez y G. Urquiza-Beltrán

Instituto Mexicano del Petróleo (IMP) [en línea]. Perspectivas de la investigación y desarrollo tecnológico del sector petrolífero al año 2025, 2001. Disponible en: www.imp.mx/investigación.

Johnk C.B. and Abdulally I.F. Experience firing petroleum Coke-An update. Foster Wheeler Energy International, Inc. 1996,

Mahagaokar U. and Hauser N. Gasification of petroleum coke in the shell coal gasification process. Fifth International Power Generation Exibition \& Conference, POWER-GEN, (5o, November 1992 , Orange Country C. Orlando Florida), 1992, pp. 17-19.

Millán F. y Julio J. La combustión de coque de petróleo en un combustor experimental de lecho fluidizado. Congreso Internacional de Calderas y Recipientes a Presión (XV, 1992, Guadalajara, Jalisco) Instituto de Investigaciones Eléctricas, Depto. de Ingeniería. Mecánica , México. Noviembre 1992.

Nagpal S., Sarkar T.K. and Sen P. K. Simulation of petcoke gasification in slagging moving bed reactors. Fuel processing Technology, ELSEVIER, pp. 617-640, 2004.
Northridge-Phillips J. A study of the Off-design performance of integrated coal gasification combined-cycle power plants. Thesis (Ph. D.), University de Stanford, 1986.

Secretaría de Energía. Oportunidades de gasificación de coque de petróleo. Instituto Mexicano del Petróleo. 2001.Secretaría de Energía [en línea]. Publicaciones 2005, Prospectiva petrolíferos 1993-2003. Disponible en: www.energía. gob.mx.

Secretaría de Energía. Prospectiva del sector eléctrico 2004-2013. Dirección General de Planeación Energética. 2003.

Secretaría de Energía [en línea]. Publicaciones 2005, Prospectiva petrolíferos 2004-2013. Disponible en: www.energía.gob.mx.

Secretaría de Energía. Una perspectiva general de la energía en México. Secretaría de energía. 2005.

www.energía.gob.mx/work/resources/localCon tent/2354petrocrudo090305.xls.

Swain J.E. Major growth in coke production taker place. Oil and Gas Journal. May 1991.

Yañez F. Petcoke: Combustible o desecho. Potencia 6.3, mayo-junio2000, pp. 38-40.

\section{Semblanza de los autores}

José Clemente González-Rocha. Es doctor en ciencias de la ingeniería mecánica por el Centro Nacional de Investigación y Desarrollo Tecnológico (CENIDET). Desde 1991, ha trabajado como profesor titular para la DGEST y ha desarrollado proyectos de investigación en el campo de termo-fluidos y plantas de potencia. Actualmente, pertenece a la División de Posgrado e Investigación del Instituto Tecnológico de Cd. Madero (I. T. C. M).

Rigoberto Longoria-Ramírez. Es doctor por la Universidad de Aberdeen, Escocia, Gran Bretaña. Ha trabajado en proyectos de investigación para el Instituto de Investigaciones Eléctricas (IIE), el centro de Ciencias de la Atmósfera de la UNAM y el Instituto Nacional de Ecología en campos relacionados con procesos químicos, contaminación ambiental y aprovechamiento de fuentes alternas de energía. Pertenece al Sistema Nacional de Investigadores (SNI) desde el año de 1989 y actualmente es profesor en el Centro de Investigación y Desarrollo Tecnológico (CENIDET).

Gustavo Urquiza-Beltrán. Es doctor en ingeniería mecánica por la Escuela Politécnica de Montreal, Canadá en 1995. Desde 1985 ha trabajado en proyectos de investigación relacionados con termo-hidráulica y turbomaquinaria en el Instituto de Investigaciones Eléctricas, en el Centro de Investigación y Desarrollo Tecnológico (CENIDET) y en el Centro de Investigación en Ingeniería y Ciencias Aplicadas (CIICAp) de la Universidad Autónoma del Estado de Morelos. Actualmente es director del CIICAp y pertenece al Sistema Nacional de Investigadores (SNI). 